\title{
Relationship between nonalcoholic steatohepatitis, PNPLA3 I148M genotype and bone mineral density in adolescents
}

Antonella Mosca ${ }^{1 *}$, MD, antonella.mosca@,opbg.net

Danilo Fintini ${ }^{2 *}$, MD, danilo.fintini@,opbg.net

Elenora Scorletti ${ }^{3,4}$, MD, E.Scorletti@,soton.ac.uk

Marco Cappa ${ }^{2}, \mathrm{MD}$, marco.cappa@opbg.net

Luisa Paone ${ }^{2}$, MD, luisa.paone@opbg.net

Anna Maria Zicari ${ }^{5}, \mathrm{MD}$, annamaria.zicari@uniroma1.it

Valerio Nobili ${ }^{1,5}, \underline{\text { nobili66@yahoo.it }}$

Christopher D Byrne ${ }^{3,4}$, C.D.Byrne@soton.ac.uk

1. Hepatology, Gastroenterology and Nutrition Unit, IRCCS "Bambino Gesù" Children's Hospital, Rome, Italy;

2. Endocrinology Unit, Pediatric University Department, Bambino Gesù Children's Hospital, Research Institute,

3. Human Development and Health Academic Unit, Faculty of Medicine, University of Southampton, Southampton, UK;

4. National Institute for Health Research Southampton Biomedical Research Centre University of Southampton, University Hospital Southampton National Health Service (NHS) Foundation Trust, Southampton, UK.

5. Department of Pediatric - University "La Sapienza", Rome, Italy

* These authors contributed equally to this work.

An electronic word count for main body of manuscript $=4440$

Number of figures and tables: 6 tables plus 3 Supplementary Table

References: 29

Word count 4400 (including 6 tables): 
Address correspondence to: Prof. Valerio Nobili, MD

Head of Hepatology, Gastroenterology and Nutrition Unit, "Bambino Gesù"

Children's Hospital IRCCS (Instituto di Ricovero e Cura a Carattere Scientifico), P.le S. Onofrio, 4 - 00165, Rome, Italy, Phone/Fax: +39-06-68592192, e-mail: nobili66@yahoo.it 


\begin{abstract}
Background and aims. It is uncertain whether non-alcoholic steatohepatitis (NASH) is a risk factor for low bone mineral density (BMD). Our aim was to investigate: a) associations between NASH and BMD values and b) associations between PNPLA3 I148M genotypes and BMD, in children with histologically-proven NAFLD.

Methods. Bone mineral density (BMD) area $(\mathrm{g} / \mathrm{cm} 2)$ was measured using dual-energy X-ray absorptiometry (DEXA). NASH was diagnosed by a Steatosis, Activity and Fibrosis (SAF) score and FLIP algorithm. Genotyping for patatin like phospholipase domain containing-3 (PNPLA3) I148M genotype (rs738409) (CC, CG, and GG) was undertaken using the TaqMan SNP genotyping allelic discrimination method. Logistic regression was used to test associations [OR (95\%CIs)] between low BMD, and both NASH and PNPLA3 I148M genotypes.

Results. 34 adolescents (mean age $13.8 \pm 1.1$ years) with histologically confirmed NAFLD were

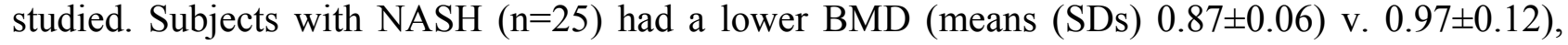
$\mathrm{p}=0.005$ ), compared to subjects without NASH. Subjects with PNPLA3 CG+GG genotypes had a lower BMD compared with subjects with PNPLA3-CC genotype (means (SDs) $0.79 \pm 0.20 \mathrm{v}$. $0.92 \pm 0.10, \mathrm{p}=0.009)$. PNPLA3 $\mathrm{CG}+\mathrm{GG}$ genotypes were independently associated with NASH [OR $(95 \%$ CIs $1.78,1.24,2.99)]$ and low BMD was associated with both PNPLA3 CG+GG (OR 3.62 (95\%CIs 1.21, 5.53), $\mathrm{p}=0.028)$ and with SAF score (OR 2.76 (95\%CIs 1.12, 5.41), $\mathrm{p}=0.045)$.

Conclusions. Taken together the independent associations between: a) low BMD and PNPLA3 $\mathrm{CG}+\mathrm{GG}$ genotype; b) low BMD and NASH; and c) PNPLA3 $\mathrm{CG}+\mathrm{GG}$ genotype and NASH, provide support for a causal relationship between NASH and low BMD.
\end{abstract}

List of abbreviations: $\mathrm{NASH}=$ nonalcoholic steatohepatitis; $\mathrm{NAFLD}=$ nonalcoholic fatty liver disease; $\mathrm{BF}=$ breastfed; PNPLA3 = Patatin like phospholipase domain containing 3; $\mathrm{BMD}=$ bone mineral density; IR= Insulin-resistance; assessed by the HOMA-IR=homeostatic model assessment; $\mathrm{NAS}=\mathrm{NAFLD}$ activity score; $\mathrm{DEXA}=$ dual-energy $\mathrm{X}$-rays absorptiometry; $\mathrm{BMI}=$ body mass index; $\mathrm{HDL}=$ high density lipoproteins; $\mathrm{LDL}=$ low density lipoproteins; $\mathrm{TG}=$ Triglycerides; AST=aspartate-aminotransferase; $\quad$ ALT=alanine-aminotransferases; $\quad$ GGT=gamma-glutamyltranspeptidase; $\mathrm{INR}=$ international normalized ratio; HOMA-IR= homeostatic model assessment of insulin resistance; $\mathrm{SAF}=$ Steatosis, Activity and Fibrosis;

Conflict of interest: All authors declare that there is no conflict of interest that could be perceived as affecting the impartiality of the reported research. 
A Statement of Financial support: No external funding supported the research described in this manuscript. This research did not receive any specific grant from any funding agency in the public, commercial or not-for-profit sector.

Authors contribution: Profs Byrne and Nobili conceptualized and designed the study. All authors approved the final manuscript as submitted and agree to be accountable for all aspects of the work.

Word count of the abstract: 247

Key Words: NASH, children, PNPLA3, BMD

\section{Key points box:}

1. Recently, it has been shown that the z score of BMD is associated with NASH in adults and NAFLD in children

2. We show that Children with NASH had a lower BMD and BMD z-score compared to subjects without NASH.

3. Children with PNPLA3 CG+GG or GG genotypes had a lower BMD and lower BMD zscore compared with subjects with PNPLA3 CC genotype. 


\section{Introduction}

Nonalcoholic fatty liver disease (NAFLD) is the leading cause of chronic liver disease in children in industrialized countries, in line with a marked increase in childhood obesity.

NAFLD includes a spectrum of diseases associated with hepatic fat, ranging from "simple hepatic steatosis", ie the accumulation of fat in more than $5 \%$ of hepatocytes, to " nonalcoholic steatohepatitis" (NASH), characterized by liver inflammation and fibrosis [1].

NAFLD is an independent risk factor for type 2 diabetes and cardiovascular disease [2-5] and emerging evidence suggests that NAFLD may influence bone density in adults, since the liver produces factors that affect bone health [ 5]. Previous evidence also suggests that a more severe liver disease in NAFLD may be associated with more pronounced reductions in bone density, since a recent study of 231 adult patients with NAFLD showed that liver fibrosis, (assessed by transient elastography), was independently associated with low bone mineral density (BMD), that is, a picture of osteoporosis and / or osteopenia (BMD z-score $<-2.5$ or $-2.5<$ BMD z-score $<-1$ ) [6].

The pathophysiological mechanisms that link NAFLD and osteoporosis or osteopenia are not fully understood. Inflammation, insulin resistance, and vitamin D deficiency are thought to mediate a link between the liver in NAFLD and alteration of bone metabolism [7,8]. That said, evidence of an association between NAFLD and osteopenia or bone density / metabolism remains inconclusive. Furthermore, it is not clear whether there is a link between NAFLD and bone structure or metabolism in children. To date, one study has suggested that children with obesity and NAFLD have decreased bone mineral density and that bone density could be further reduced in children with NASH [9]. However, it is not clear from this study whether there is a causal relationship between NASH and low mineral density.

Since it remains uncertain whether NASH is associated with low bone mineral density and if so, any link between liver disease and low bone mineral density could be a causal relationship. At the 
same time, it is known that the I148M genotype (ns738409) of the containing phospholipase containing 3 domain (PNPLA3) is associated with severe liver disease in NAFLD. Therefore, our objective was to investigate the associations between NASH and BMD in children who had assessed the severity of NAFLD according to histological staging criteria, and in which genotype data were also available [10]. We hypothesized, in a proof of concept study, that any association between the PNLA3 CG + GG genotype (or the GG genotype alone) and the low BMD would provide evidence to support the notion of a causal relationship between NAFLD and low BMD. 


\section{Patients and Methods}

In this proof of concept study, 34 obese adolescents (age range, 11 to 16.8 years) with biopsyproven NAFLD were included. A diagnosis of NAFLD was established by excluding the viral infections (serological dosage of HAV, HBV, HCV, Cytomegalovirus and Epstein-Barr virus), the alcohol intake and the use of drugs such as prednisone, valproate. Furthermore, patients with autoimmune or metabolic liver diseases, alpha-1-antitrypsin deficiency, Wilson's disease, celiac disease, genetic syndromes, diabetes or genetic dyslipidaemia were also excluded, in according with position paper of ESPGHAN committee (Differential diagnosis should be based first on clinical features, then on blood tests, and finally liver biopsy must be considered). The patients included in the study were selected from 180 consecutive obese patients with liver steatosis admitted at the Hepato-Metabolic Unit of Bambino Gesù Children's Hospital between September 2016 and December 2017. The inclusion criteria for the study were: a biopsy-proven diagnosis of NAFLD, subjects were aged between 12 and 17, there was no specific dietary or other therapeutic treatment. 96 patients were excluded from the study because of: age $<11$ years, pubertal stage (Tanner stage $<$ 1); presence of comorbidities (OSAS severe, hypertension, osteopenia); medication (such as metformin, steroids and vitamin D3); adherence to a diet or to dietary supplements. Of the remaining 84 subjects, only 43 were eligible for liver biopsy, according to ESPGHAN. In six patients other liver diseases besides NAFLD were confirmed. Of the remaining 37 patients, 3 refused to participate in the study.

Clinical and experimental data were acquired at diagnosis in all patients. The study protocol was approved by the Ethical Committee of Bambino Gesù Children's Hospital and written informed consent was obtained from the parents of the children.

\section{Anthropometrical and Serum biomarkers}

The body mass index (BMI) - Weight $(\mathrm{kg}) /$ height $^{2}$ and waist circumference $(\mathrm{cm})$ were measured. CDC percentiles were used to define overweight $\left(>85^{\text {th }}\right.$ and $<95^{\text {th }}$ centile) and obese $\left(>95^{\text {th }}\right.$ centile) for adolescents[11]. Tanner's tables were used to define pubertal stage [12]. Serum glucose, 
triglycerides, cholesterol-total, high-density lipoprotein (HDL) cholesterol and low-density lipoprotein (LDL)], aspartate- (AST) and alanine- (ALT) aminotransferases, gamma-glutamyltranspeptidase (GGT), fasting plasma glucose and insulin were measured in all patients after an overnight 12-h fast. In all patients Oral Glucose Tolerance Tests (OGTT) were performed during the primary evaluation. Insulin-resistance (IR) was assessed by the homeostatic model assessment $($ HOMA $)[$ HOMA-IR $=($ insulin $(\mu \mathrm{IU} / \mathrm{ml}) \times$ glucose $(\mathrm{mmol} / \mathrm{l})) / 22.5)]$. A cut-off value of $>2.5$ was considered as an index of insulin resistance[13].

In all patients, serum 25-hydroxyvitamin $\mathrm{D}$ [25(OH)D, vitamin $\mathrm{D}]$ concentration was measured by radioimmunoassay (IDS Immunodiagnostics, IDS Limited, Tyne and Wear, UK). Patients with vitamin D values $<20 \mathrm{ng} / \mathrm{mL}$ were excluded [14].

\section{Bone mineral density measurement}

The bone mineral density (BMD) area $(\mathrm{g} / \mathrm{cm} 2)$ was measured using dual-energy X-rays absorptiometry (DEXA) by a single authorized and trained technician. DEXA measurements were calibrated on a single standard and reference values were obtained. DEXA calibration was checked through an internal reference system and spinal column ghosts were measured every day, which are equivalent reference standards for bone and soft tissue [15]. The DEXA quality control program included technologist training and monitoring, precision evaluation and central review of all scans. BMD measurements provided absolute values for each anatomical site of lumbar spine, total hip, neck of the femur and the whole body. DEXA measurements also included Bone Mineral Content (BMC), FAT mass (grams and \%) and Lean mass [16,17].

\section{Patatin like phospholipase domain containing-3 (PNPLA3)}

Genotyping for PNPLA3 I148M gene variant (rs738409) was carried out at first diagnosis of liver involvement using the TaqMan SNP genotyping allelic discrimination method (Applied Biosystems, Foster City, CA) (cat. C_7241_10). Genotype was analyzed with SDS software v.1.3.0 
(ABI Prism 7500, Foster City, CA).

\section{Liver biopsy}

Under general anaesthesia, an echo-guided liver biopsy was undertaken using an automatic core biopsy device (Biopince, Amedic, Sweden) with an 18-G needle. Histological analysis was performed by a single pathologist blinded to clinical and laboratory data. The histological features of steatosis (0-3), lobular inflammation (0-3), and hepatocyte ballooning (0-2) and Fibrosis (0-4) according to NAFLD Activity Score (NAS)[18]. NASH was diagnosed by a Steatosis, Activity and Fibrosis (SAF) score and FLIP algorithm: severe $(=2)$, moderate $(=1)$ and mild $(=0)$. NASH was diagnosed by the presence of moderate or severe SAF $[19,20]$.

\section{Statistical Analysis}

Data were analysed using MedCalc software (version 18.2.1, 2018, Ostend, Belgium) unless otherwise stated. Data are reported as mean (SD) for normal continuous variables, median and IQR ranges for non-normally distributed continuous variables and frequencies for categorical variables. Differences between groups were evaluated with the Student's t-test for variables that were normally distributed or with the Mann-Whitney $U$ test if variables were non-normally distributed and with ANOVA. For percentages, differences between groups were assessed with the $\chi 2$ test or with the $\chi 2$ test for trend as appropriate. Binary logistic regression was used to test associations (ORs, 95\%CIs) between: a) NASH v no NASH as the outcome and b) low v. high BMD as the outcome, with PNPLA3 I148M genotypes, SAF score, and metabolic parameters as explanatory variables in the models. Models were adjusted for age, BMI and fat mass. Statistical significance was defined as $\mathrm{p}<0.05$. 


\section{Results}

Thirty-four adolescents, age range 12-17 years, with biopsy-proven NAFLD were included in the study. Table 1 shows the anthropometric and biochemical characteristics of the subjects.

Supplementary Table 1 (A, B) shows the histological diagnosis of NASH in our population, according to the fatty liver inhibition of progression-FLIP- algorithm and the steatosis, activity, fibrosis -SAF- score. 25/34 subjects had NASH. The differences between groups according to mild, moderate and severe SAF scores are shown in Table 2. As expected there were between group differences for HOMA-IR with higher levels of HOMA-IR in the more severe liver disease groups. BMD was also lower in the more severe liver disease groups.

Table 3 shows the between SAF group differences for regional BMDs in subjects according to mild, moderate and severe SAF scores. There were between group differences in the BMDs of lumbar vertebrae $(p=0.03)$, hip $(p=0.04)$ and total BMD $(p=0.002)$. Supplementary table 2 shows the between SAF group differences for BMC, fat mass, lean mass and BMD z-scores. There were between group differences for BMD z-scores $(p=0.048)$.

Table 4 shows the differences in age, BMI, waist circumference and DEXA parameters according to NASH status (NASH v. no NASH) and PNPLA3 I148M genotypes (PNPLA3 CG+GG v. CC). Subjects with NASH had a lower BMD and BMD z-score compared to subjects without NASH. Subjects with PNPLA3 CG+GG genotypes had a lower BMD and lower BMD z-score compared with subjects with PNPLA3 CC genotype. There were 7 subjects with GG genotype, and we therefore tested whether there were differences in age, BMI, waist circumference and DEXA parameters after stratifying the patients according to the PNPLA3 GG v. GC+CC genotypes. Consistent with the prior stratification (i.e. PNPLA3 CG+GG v. CC), BMD and BMD z-score were both lower in subjects with PNPLA3 GG v. GC+CC genotypes (BMDs 0.75 (0.17) v. 0.98 (0.15), $\mathrm{p}=0.03$ and $(\mathrm{BMD} z$-scores $0.38(0.17)$ v. $0.88(0.27), \mathrm{p}=0.007$. 
Next we tested what factors were independently associated with NASH, and specifically whether PNPLA3 CG+GG genotype and BMD were both independently associated with NASH. Adjusting for age, BMI and total body fat mass (grammes), low BMD and PNPLA3 CG+GG were both independently associated with NASH (Table 5). Similar results were obtained if genotypes were stratified according to PNPLA3 GG v. PNPLA3 CG+CC. In this analysis, adjusting for age, BMI and total body fat mass (grammes), PNPLA3 GG was independently associated with NASH (OR $2.82(1.38,4.77), \mathrm{p}=0.02)$.

Finally, we investigated associations between low BMD (stratified by the median BMD value) as the outcome, and SAF score, PNPLA3 I148M genotype and metabolic parameters as exposures (Table 6). Adjusting for age, BMI and total body fat mass (grammes), SAF score (OR 2.76 (95\%CIs 1.12, 5.41), $\mathrm{p}=0.045)$ and PNPLA3 CG+GG genotypes (OR 3.62 (95\%CIs 1.21, 5.53), $\mathrm{p}=0.028$ ) were both associated with low BMD. Similar results were again obtained if genotypes were stratified according to PNPLA3 GG v. PNPLA3 CG+CC. In this analysis, adjusting for age, BMI and total body fat mass (grammes), PNPLA3 GG was independently associated with low BMD (OR 4.18 (1.57, 6.22), $\mathrm{p}=0.01)$. 


\section{Discussion}

The novel results of our study show that subjects with NASH had a lower BMD and BMD z-score compared to subjects without NASH.

Furthermore, subjects with PNPLA3 CG+GG genotypes had a lower BMD and lower BMD z-score compared to subjects with PNPLA3 CC genotype. BMD was independently associated with NASH and both SAF score and PNPLA3 CG+GG genotype were independently associated with low BMD. It is important to underline that, since the homozygous PNPLA3 genotype is even more strongly associated with a more severe liver disease in NAFLD, compared to the heterozygous genotype [21], although there were only seven subjects with GG genotype, consistent results were obtained for the GG genotype (comparing CG + GG genotypes) even when stratified by genotypes was GG v. $\mathrm{CG}+\mathrm{CC}$.

The pathophysiological mechanisms linking NASH and low BMD are not fully understood. Potential pathogenic mediators linking liver and bone, include low levels of vitamin D, fibronectin, IGF-1, the RANKL / OPG system, TNF- $\alpha$ and IL-6, and all may play important roles in the pathogenesis of bone loss in chronic liver disease [22]. In our study, subjects with low levels of vitamin D were excluded. Although many subjects with chronic liver disease have low levels of vitamin D [14], these authors failed to show any significant correlation between BMD and vitamin D levels $[23,24]$.

Liver lipid accumulation, reactive oxidative stress and activated liver macrophages (that release pro-inflammatory cytokines) could all modulate systemic inflammatory immune responses and theoretically influence bone health [25]. Hepatic inflammation and fibrosis are also linked to adipose inflammation and insulin resistance, through the systemic release of inflammatory mediators such as TNF- $\alpha$, IL-6 and chemoattractant monocytes protein-1 (MCP-1) from adipose tissue, and these mediators also potentially influence bone remodelling [25,26]. In fact, it is known that the MCP-1 produced by osteoblasts and osteocytes, producing RANKL, stimulates the 
expression of $\mathrm{MCP}-1$ receptors on osteoclasts, thus altering the bone metabolism. In addition, parathormone (PTH), IL-1, TNF- $\alpha$ and vitamin D3 indirectly regulate the activation of osteoclasts [27-29]. Therefore, our results showing a strong independent association between low bone mineral density and insulin resistance and with the diagnosis of NASH (table 6), confirm that insulin resistance is one of the primum movens of the production of inflammation mediators. which induce not only the progression of hepatic injury but also the activation of osteoclastogenesis and the suppression of osteoblasts at the level of the bone matrix.

In support of these associations we describe, we have also shown that the PNPLA3 I148M CG + GG (or GG alone) genotype is also independently associated with low bone mineral density, providing further support for the notion that hepatic disease per se it could affect bone density and not vice versa.

This proof of concept study has strengths and limitations that should be considered. To our knowledge, this is the first study to study the association between BMD and NASH assessed by liver histology that also includes genotypic data, with a genotype known to be associated with severity of liver disease. We are not able to comment on PTH values since this measurement was not undertaken in all subjects but we have excluded those children with vitamin D deficiency. We have also excluded pre-pubertal children assessed by Tanner charts. The study has limitations. Our study is a cross-sectional study design, and therefore it is not possible to establish the direction of the association and establish causality between NASH and low BMD. However, that said, since we observed an independent association between PNPLA3 CG+GG (or GG alone), and low BMD; and since these genotypes are known to be effectors of more severe liver disease in NAFLD (than the CC genotype), our data lend credence to the notion that NASH may cause low BMD. These data also provide evidence against the possibility of reverse causality, since low BMD could not 'cause' the genotype. 
In conclusion, our data show that subjects with NASH had a lower score of BMD and BMD z compared to subjects without NASH. Subjects with PNPLA3 CG + GG (or GG) genotypes had lower BMD and a lower BMD Z score than subjects with PNPLA3 CC genotype. Although it is possible that our results have happened by chance, we suggest that the strength of the associations we have observed means that our findings guarantee further investigation and verification in other cohorts. Therefore, we believe it is useful to investigate in obese adolescents with bone is also the bone metabolism through not only the determination of vitamin $\mathrm{D}$ values, but also PTH and possibly, especially in the presence of SNPs for NASH, subject them to DEXA examination, in order to prevent possible early clinical manifestations of osteoporosis, which has a significant impact on the patient's life and on health expenditure.

Acknowledgments. CDB is supported in part by the Southampton NIHR Biomedical Research Centre. 


\section{References}

1. Nobili V, Svegliati-Baroni G, Alisi A, Miele L, Valenti L, Vajro P. A 360-degree overview of paediatric NAFLD: recent insights. J Hepatol. 2013;58(6):1218-29. doi: 10.1016/j.jhep.2012.12.003.

2. Wójcik-Cichy K, Koślińska-Berkan E, Piekarska A. The influence of NAFLD on the risk of atherosclerosis and cardiovascular diseases. Clin Exp Hepatol. 2018;4(1):1-6. doi: 10.5114/ceh.2018.73155.

3. Mantovani A, Byrne CD, Bonora E, Targher G. Nonalcoholic Fatty Liver Disease and Risk of Incident Type 2 Diabetes: A Meta-analysis. Diabetes Care. 2018 Feb;41(2):372-382. doi: $10.2337 / \mathrm{dc} 17-1902$.

4. Byrne CD, Targher G. NAFLD: a multisystem disease. J Hepatol. 2015 Apr;62(1 Suppl):S47-64. doi: 10.1016/j.jhep.2014.12.012.

5. Targher G, Byrne CD, Lonardo A, Zoppini G, Barbui C. Non-alcoholic fatty liver disease and risk of incident cardiovascular disease: A meta-analysis. J Hepatol.2016 Sep;65(3):589600. doi: 10.1016/j.jhep.2016.05.013

6. Kim G, Kim KJ, Rhee Y, Lim SK. Significant liver fibrosis assessed using liver transient elastography is independently associated with low bone mineral density in patients with non-alcoholic fatty liver disease. PLoS One. 2017;12(7):e0182202. doi: 10.1371/journal.pone.0182202.

7. Yadav A, Carey EJ. Osteoporosis in chronic liver disease. Nutr Clin Pract. 2013; 28:52-64

8. Gatta A, Verardo A, Di Pascoli M, Giannini S, Bolognesi M. Hepatic osteodystrophy. Clin Cases Miner Bone Metab. 2014; 11:185-91.

9. Pardee PE, Dunn W, Schwimmer JB. Non-alcoholic fatty liver disease is associated with low bone mineral density in obese children. Aliment Pharmacol Ther. 2012;35(2):248-54. doi: 10.1111/j.1365-2036.2011.04924.x. 
10. Del Campo JA, Gallego-Durán R, Gallego P, Grande L. Genetic and Epigenetic Regulation in Nonalcoholic Fatty Liver Disease (NAFLD). Int J Mol Sci. 2018;19(3). pii: E911. doi: 10.3390/ijms19030911

11. Growth Charts - $2000 \quad$ CDC Growth Charts https://www.cdc.gov/growthcharts/cdc charts.htm

12. Tanner JM. Normal growth and techniques of growth assessment. Clin Endocrinol Metab. 1986;15(3):411-51. Review

13. Tang $\mathrm{Q}$, Li $\mathrm{X}$, Song $\mathrm{P}, \mathrm{Xu} \mathrm{L}$. Optimal cut-off values for the homeostasis model assessment of insulin resistance (HOMA-IR) and pre-diabetes screening: Developments in research and prospects for the future. Drug Discov Ther. 2015;9(6):380-5. doi: $10.5582 /$ ddt.2015.01207

14. Della Corte C, Carpino G, De Vito R et al. Docosahexanoic Acid Plus Vitamin D Treatment Improves Features of NAFLD in Children with Serum Vitamin D Deficiency: Results from a Single Centre Trial.PLoS One. 2016 Dec 15;11(12):e0168216. doi: 10.1371/journal.pone.0168216

15. Martin-Calvo N, Moreno-Galarraga L, Martinez-Gonzalez MA. Association between Body Mass Index, Waist-to-Height Ratio and Adiposity in Children: A Systematic Review and Meta-Analysis. Nutrients. 2016 Aug 20;8(8). pii: E512. doi: 10.3390/nu8080512.

16. Binkley N, Kiebzak GM, Lewiecki EM et al. Recalculation of the NHANES database SD improves T-score agreement and reduces osteoporosis prevalence. J Bone Miner Res. $2005 ; 20: 195-201$.

17. Kanis JA, McCloskey EV, Johansson H et al. A reference standard for the description of osteoporosis. Bone. 2008; 42:467-75.

18. Santiago-Rolón A, Purcell D, Rosado K, Toro DH. A Comparison of Brunt's Criteria, the Non-Alcoholic Fatty Liver Disease Activity Score (NAS), and a Proposed NAS Scoring that Includes Fibrosis in Non-Alcoholic Fatty Liver Disease Staging. P R Health Sci J. 2015 Dec;34(4):189-94

19. Bedossa P. Diagnosis of non-alcoholic fatty liver disease/non-alcoholic steatohepatitis: Why liver biopsy is essential. Liver Int. 2018 Feb;38 Suppl 1:64-66. doi: 10.1111/liv.13653

20. Bedossa P; FLIP Pathology Consortium. Utility and appropriateness of the fatty liver inhibition of progression (FLIP) algorithm and steatosis, activity, and fibrosis (SAF) score in the evaluation of biopsies of nonalcoholic fatty liver disease. Hepatology. 2014 Aug;60(2):565-75. doi: 10.1002/hep.27173. 
21. Salameh H, Hanayneh MA, Masadeh M et al. PNPLA3 as a Genetic Determinant of Risk for and Severity of Non-alcoholic Fatty Liver Disease Spectrum. J Clin Transl Hepatol. 2016 Sep 28;4(3):175-191

22. Mann JP, De Vito R, Mosca A, et al. Portal inflammation is independently associated with fibrosis and metabolic syndrome in pediatric nonalcoholic fatty liver disease. Hepatology. 2016 Mar;63(3):745-53. doi: 10.1002/hep.28374.

23. Targher G, Lonardo A, Rossini M. Nonalcoholic fatty liver disease and decreased bone mineral density: is there a link? J Endocrinol Investig 2015, 38:817-825.

24. Lonardo A, Ballestri S, Marchesini G, et al. Nonalcoholic fatty liver disease: a precursor of the metabolic syndrome. Digestive liver Disease: Official J Italian Soc Gastroenterol Italian Assoc Study Liver, 2015: 47:181-190.

25. Graves DT, Jiang Y, Valente AJ. Regulated expression of MCP-1 by osteoblastic cells in vitro and in vivo. Histol Histopathol. 1999;14:1347-54.

26. Ha H, Kwak HB, Lee SW, et al. Reactive oxygen species mediate RANK signaling in osteoclasts. Exp Cell Res. 2004;301:119-27

27. Post TM, Cremers SC, Kerbusch T, Danhof M. Bone physiology, disease and treatment: towards disease system analysis in osteoporosis. Clin Pharmacokinet. 2010;49:89-118

28. Powell WF Jr, Barry KJ, Tulum I, et al. Targeted ablation of the PTH/PTHrP receptor in osteocytes impairs bone structure and homeostatic calcemic responses. J Endocrinol. 2011;209:21-32.

29. Stolk RP, Van Daele PL, Pols HA et al. Hyperinsulinemia and bone mineral density in an elderly population: the Rotterdam Study. Bone, 1996; 18:545-549 
